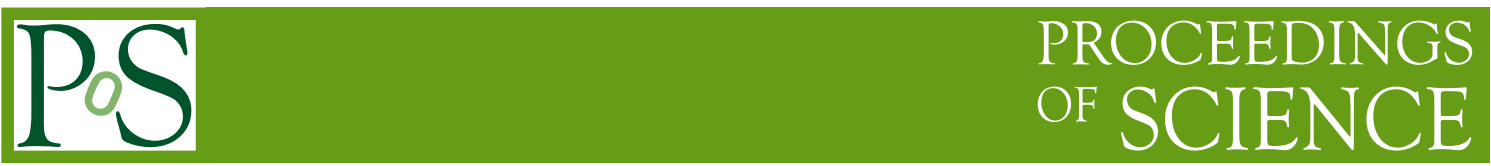

\title{
Measuring radiative capture rates at DRAGON
}

\author{
U. Hager ${ }^{*}{ }^{a}$ L. Buchmann, ${ }^{b}$ B. Davids, ${ }^{b}$ J. Fallis, ${ }^{b}$ U. Greife, ${ }^{a}$ D.A. Hutcheon, ${ }^{b}$ \\ D. Ottewell, ${ }^{b}$ A. Rojas, ${ }^{b}$ C. Ruiz ${ }^{b}$
}

${ }^{a}$ Colorado School of Mines, Golden, CO, USA

${ }^{b}$ TRIUMF, Vancouver, Canada V6T $2 A 3$

E-mail: uhageremines.edu

\begin{abstract}
The DRAGON recoil separator facility is located at the ISAC facility at TRIUMF, Vancouver. It is designed to measure radiative alpha and proton capture reactions of astrophysical importance in inverse kinematics. The recently installed Supernanogan ion source at ISAC provides stable beams of high intensities. The DRAGON collaboration has taken advantage of this over the last years by measuring several reactions requiring high-intensity stable oxygen beams. In particular,the ${ }^{17} \mathrm{O}(p, \gamma){ }^{18} \mathrm{~F}$ and ${ }^{16} \mathrm{O}(\alpha, \gamma){ }^{20} \mathrm{Ne}$ reaction rates were recently measured. The former reaction is part of the hot $\mathrm{CNO}$ cycle, and strongly influences the abundance of ${ }^{18} \mathrm{~F}$ in classical novae. Because of its relatively long lifetime, ${ }^{18} \mathrm{~F}$ is a possible target for satellite-based gamma-ray spectroscopy. The ${ }^{16} \mathrm{O}(\alpha, \gamma)^{20} \mathrm{Ne}$ reaction plays a role in steady-state helium burning in massive stars, where it follows the ${ }^{12} \mathrm{C}(\alpha, \gamma)$ reaction. At astrophysically relevant energies, the reaction proceeds exclusively via direct capture, resulting in a low rate. In both cases, the unique capabilities of DRAGON enabled determination not only of the total reaction rates, but also of decay branching ratios. Results from both experiments will be presented.
\end{abstract}

XII International Symposium on Nuclei in the Cosmos,

August 5-12, 2012

Cairns, Australia

\footnotetext{
* Speaker.
} 


\section{The DRAGON recoil separator}

The DRAGON recoil separator [1] is located at the ISAC facility at TRIUMF, Vancouver, Canada. It is designed to measure reaction cross sections in inverse kinematics. A schematic view of the DRAGON facility is shown in Fig. 1. The DRAGON windowless gas target is filled with

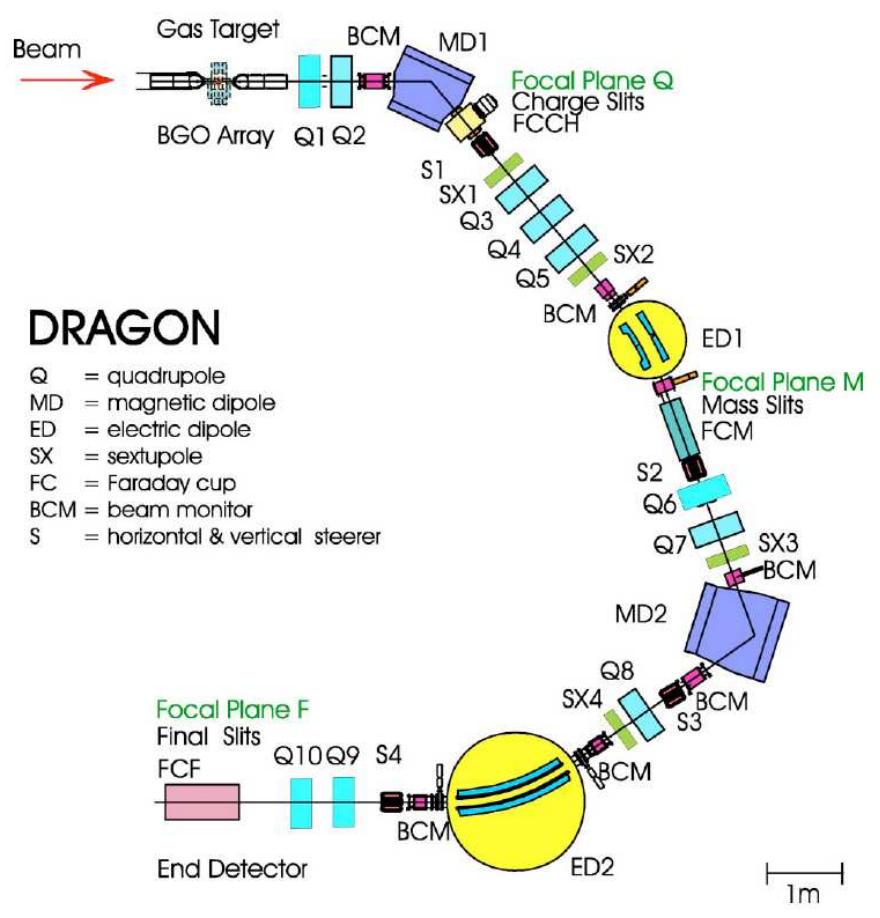

Figure 1: Schematic view of the DRAGON facility.

either ${ }^{4} \mathrm{He}$ or $\mathrm{H}_{2}$; the effective target length is $12.3(5) \mathrm{cm}$ [1]. The DRAGON target is surrounded by an array of 30 bismuth germanate (BGO) $\gamma$-ray detectors in a tight geometry. In order to suppress background, the recoil ions produced in the reaction of interest are usually detected in coincidence with $\gamma$ events in the BGO array, after separation from the beam using a two-stage electromagnetic separator with an angular acceptance of $21 \mathrm{mrad}$ for reactions at the center of the target. At the focal plane, an ionization chamber (IC) [2] is used to detect recoils from the reaction. Its segmented anode enabled stopping power measurements and thus particle identification (PID). Alternatively, a double-sided silicon strip detector (DSSSD) can be used enabling determination of the position. In addition, two MCPs (micro-channel plates) upstream of the ionization chamber are used to measure the time-of-flight (TOF) across the $59 \mathrm{~cm}$ distance between them, and the time-of-flight through the separator relative to the $\gamma$ event [3].

\section{2. ${ }^{16} \mathbf{O}(\alpha, \gamma){ }^{20} \mathrm{Ne}$}

The first reaction of the stellar helium burning stage is the triple- $\alpha$ process, producing ${ }^{12} \mathrm{C}$. After the production of ${ }^{12} \mathrm{C}$, other $\alpha$-cluster nuclei can in principle be produced. At typical helium burning temperatures of $1-3 \times 10^{8} \mathrm{~K}$ (effective energy for helium burning reaction $E_{c m} \approx$ 
$0.3 \mathrm{MeV}$ ), their production is, however, hindered by the increasing Coulomb barriers, and by the low ${ }^{16} \mathrm{O}(\alpha, \gamma)^{20} \mathrm{Ne}$ rate [4]. Thus, two of the main end products of steady state helium burning are ${ }^{12} \mathrm{C}$ and ${ }^{16} \mathrm{O}$, with their ratio determined in part by the ${ }^{16} \mathrm{O}(\alpha, \gamma){ }^{20} \mathrm{Ne}$ rate. This ratio also determines the abundances of the products of later carbon burning, mainly ${ }^{20} \mathrm{Ne},{ }^{23} \mathrm{Na},{ }^{23,24} \mathrm{Mg}$ and ${ }^{28} \mathrm{Si}$, compared to the products of oxygen burning like aluminium [4].

The reason for the low ${ }^{16} \mathrm{O}(\alpha, \gamma){ }^{20} \mathrm{Ne}$ rate at helium burning temperatures is the absence of an excited state in ${ }^{20} \mathrm{Ne}$ in the relevant energy region. The reaction therefore proceeds mainly via non-resonant direct capture (DC).

The DRAGON group initially performed a direct measurement of the ${ }^{16} \mathrm{O}(\alpha, \gamma)^{20} \mathrm{Ne}$ cross section at $E_{\mathrm{c} . \mathrm{m} .}=2.26 \mathrm{MeV}$ [5], sufficiently far from the tails of the higher $J^{\pi}=3^{-}$and the lower $0^{+}$resonances for the DC to dominate the reaction. Additional measurements were then performed at $E_{\text {c.m. }}=1.69 \mathrm{MeV}$, between the $1^{-}$resonance at $E_{x}=5788 \mathrm{MeV}$ and the $0^{+}$resonance at $E_{x}=6725 \mathrm{MeV}$. This energy was chosen to minimize resonant contributions, while still having a sufficiently high rate to enable determination of the relative strengths of capture to the ground state $\left(S_{0}\right)$ and to the first excited state $\left(S_{2}\right)$. In addition, a measurement of the ${ }^{16} \mathrm{O}(\alpha, \gamma){ }^{20} \mathrm{Ne}$ cross section at the $E_{\mathrm{c} . \mathrm{m}}=2.43 \mathrm{MeV}$ resonance was performed and the branching ratios and resonance strength determined. Another measurement was done at $E_{\text {c.m. }}=2.35 \mathrm{MeV}$, on the low-energy edge of the $E_{\mathrm{c} . \mathrm{m} .}=2.43 \mathrm{MeV}$ resonance. These data points constitute an experimental test of the recent comprehensive $R$-matrix predictions published in [6]. Figure 2 compares the results obtained at DRAGON to previous measurements. Good agreement is found for $S_{0}$ with the previous measurement by Hahn et al. [7] at this energy. Our value for the total $S$ factor also agrees well with the $R$-matrix calculation performed by Costantini et al. [6].

\section{3. ${ }^{17} \mathbf{O}(p, \gamma){ }^{18} \mathbf{F}$}

One of the goals of the satellite-based $\gamma$-astronomy is to observe the emission of $511 \mathrm{keV} \gamma$ radiation from classical novae. This emission is produced by the electron-positron annihilation following the $\beta^{+}$decay of radioactive nuclei in the expanding envelope [10]. ${ }^{18} \mathrm{~F}$ is the most important contributor, since its half-life of 110 min means that it is still undergoing $\beta$-decay when the envelope becomes transparent to $\gamma$-rays.

${ }^{18} \mathrm{~F}$ nucleosynthesis in classical novae strongly depends on the thermonuclear rate of ${ }^{17} \mathrm{O}(p, \gamma){ }^{18} \mathrm{~F}$ [11], which is part of the hot CNO cycle [4]. The relevant temperature range is between 0.1 and 0.4 GK [12]. Using a hydrodynamic nova model with a nuclear reaction network, José et al. [13] predict that ${ }^{17} \mathrm{O}$ is among the main products in the ejecta of classical novae. The amount of ${ }^{18} \mathrm{~F}$ produced depends on the ratio of the ${ }^{17} \mathrm{O}(p, \gamma){ }^{18} \mathrm{~F}$ and ${ }^{17} \mathrm{O}(p, \alpha){ }^{14} \mathrm{~N}$ rates. Iliadis et al. [14] used profiles from hydrodynamic nova simulations and varied the reaction rates within uncertainties. They found that the ${ }^{18} \mathrm{~F}$ abundance was sensitive to the reaction rate uncertainties at that time, with variations of up to a factor 100 for variations of ${ }^{18} \mathrm{~F}(p, \alpha),{ }^{17} \mathrm{O}(p, \alpha){ }^{14} \mathrm{~N}$ and ${ }^{17} \mathrm{O}(p, \gamma){ }^{18} \mathrm{~F}$.

Current literature values of the ${ }^{17} \mathrm{O}(p, \gamma){ }^{18} \mathrm{~F}$ rate give conflicting information both for the direct capture reaction and the strength of the $E_{\text {c.m. }}=183 \mathrm{keV}$ resonance.

Direct measurements of the astrophysical $S$ factor of the ${ }^{17} \mathrm{O}(p, \gamma){ }^{18} \mathrm{~F}$ reaction at four center-ofmass energies between 250 and $500 \mathrm{keV}$ were performed at DRAGON. The stable ${ }^{17} \mathrm{O}$ beam was produced by the Supernanogan off-line ECR ion source [15]. The maximum beam intensity on 


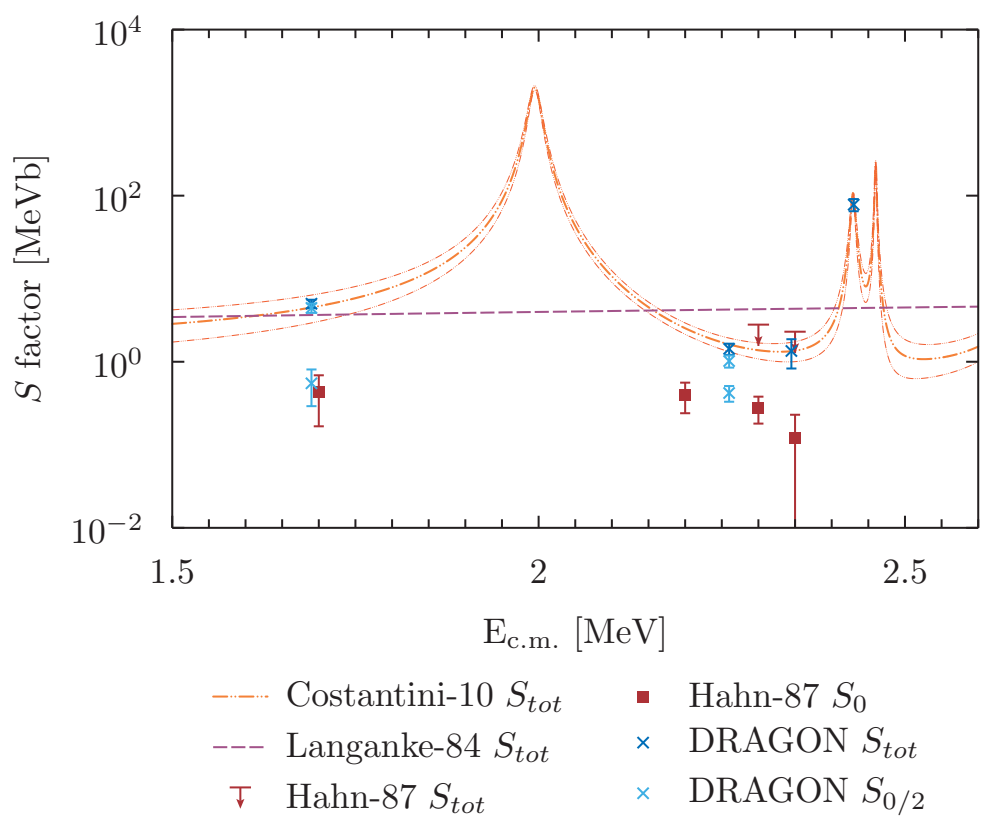

Figure 2: Comparison of this work to previous results: the total $S$ factor given by Costantini et al. [6], Langanke [8], Hahn et al. [7], and the DRAGON results from the present work and [5]. Also shown are the upper limits by Hahn et al. for $S_{0}$. The points labelled 'DRAGON $S_{0 / 2}$ ' are the results presented in [5] and [9] for $S_{0}$ (lower value) and $S_{2}$ (larger value). From [9].

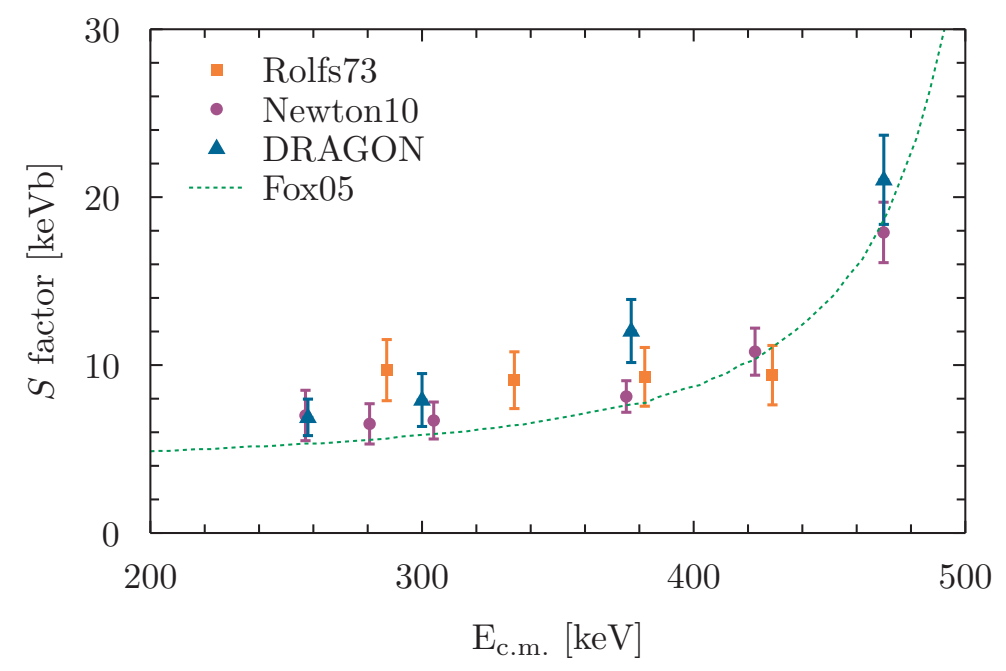

Figure 3: $S$ factor, comparison to literature [17, 18, 12]. From [16].

target was $\approx 1.5 \cdot 10^{12} \mathrm{~s}^{-1}$. Figure 3 shows the $S$ factor values determined in this work [16] as well as those measured by Rolfs [17] and Newton et al. [18]. Our results clearly show the $S$ factor decreasing with decreasing energy, in contrast to [17], where the $S$ factor was found to be constant with energy. For the three highest energies, our values lie above those given by Newton et al . [18], though the discrepancy is less than $1 \sigma$ except for the values at $E_{\text {c.m. }}=375 \mathrm{keV}$, which differ by about $2.2 \sigma$. Our values are also consistently higher than the evaluation by Fox et al. [12]. Comparing the shape of the calculated curve presented by Fox et al. to the energy dependence of 
the $S$ factor presented in this work, it seems likely that the width or strength of the $E_{\text {c.m. }}=557 \mathrm{keV}$ or $E_{\mathrm{c} . \mathrm{m} .}=677 \mathrm{keV}$ resonances used in Ref. [12] are too low and need to be re-investigated in future experiments.

\section{4. ${ }^{26 m} \mathbf{A l}(p, \gamma){ }^{27} \mathbf{S i}$}

Stellar nucleosynthesis of the cosmic $\gamma$-ray emitting nucleus ${ }^{26} \mathrm{Al}$ is complicated by the presence of a spin isomer located just above the ground state, whose $\beta$-decay bypasses the ground state and consequently, reduces the flux for the cosmic $\gamma$-ray emission. In general, the isomeric state is expected to act as an independent radioactive nucleus. However, in high temperature environments, such as ONe novae and supernovae, it is possible for the ground and isomeric states to reach a state of thermal equilibrium. As such, the creation/destruction rate of the isomer may significantly affect the synthesized abundance of the cosmic $\gamma$-ray emitting ground state. In recent work $[19,20]$ key astrophysical resonances for the ${ }^{26 m} \mathrm{Al}(p, \gamma)^{27} \mathrm{Si}$ destruction reaction were identified. In particular, $l_{p}=1$ and $l_{p}=0$ resonances at $378 \mathrm{keV}$ and $448 \mathrm{keV}$, respectively, were identified as dominating the rate in high temperature scenarios. The strength of these two, key states remain considerably uncertain. A direct measurement of the $E_{\text {c.m. }}=448 \mathrm{keV}$ resonance was performed with the use of the DRAGON recoil separator and intense ${ }^{26 m} \mathrm{Al}$ beams available at TRIUMF. The energy covered in the experiment ranged from $E_{\mathrm{c} . \mathrm{m} .}=430-470 \mathrm{keV}$, using several beam energies and target thicknesses. ${ }^{27} \mathrm{Si}$ recoils were observed at the expected resonance energy, and data analysis is currently under way to determine how many of those recoils originate from the ${ }^{26 m} \mathrm{Al}(p, \gamma)$ reaction, and how many from the ground state ${ }^{26} \mathrm{Al}(p, \gamma)$ reaction.

\section{References}

[1] D. A. Hutcheon, S. Bishop, L. Buchmann, M. L. Chatterjee, A. A. Chen, J. M. D'Auria, S. Engel, D. Gigliotti, U. Greife, D. Hunter, A. Hussein, C. C. Jewett, N. Khan, M. Lamey, A. M. Laird, W. Liu, A. Olin, D. Ottewell, J. G. Rogers, G. Roy, H. Sprenger, and C. Wrede, The DRAGON facility for nuclear astrophysics at TRIUMF-ISAC: design, construction and operation, Nucl. Instr. Meth. A 498 (2003) 190-210.

[2] C. Vockenhuber, L. Buchmann, J. Caggiano, A.A. Chen, J.M. D’Auria, C.A. Davis, U. Greife, A. Hussein, D.A. Hutcheon, D. Ottewell, C.O. Ouellet, A. Parikh, J. Pearson, C. Ruiz, G. Ruprecht, M. Trinczek, and J. Zylberberg, Improvements of the DRAGON recoil separator at ISAC, Nucl. Instr. Meth. B 266 (2008) 4167-4170.

[3] C. Vockenhuber, L.E. Erikson, L. Buchmann, U. Greife, U. Hager, D.A. Hutcheon, M. Lamey, P. Machule, D. Ottewell, C. Ruiz, and G. Ruprecht, A transmission time-of-flight system for particle identification at a recoil mass separator at low energies, Nucl. Instr. Meth. A 603 (2009) 372-378.

[4] C. E. Rolfs and W. S. Rodney. Cauldrons in the Cosmos. The University of Chicago Press 1988.

[5] U. Hager, J. R. Brown, L. Buchmann, M. Carmona-Gallardo, L. Erikson, J. Fallis, U. Greife, D. Hutcheon, D. Ottewell, C. Ruiz, S. Sjue, and C. Vockenhuber, Direct total cross section measurement of the ${ }^{16} O(\alpha, \gamma){ }^{20} \mathrm{Ne}$ reaction at $E_{\mathrm{c} . \mathrm{m} .}=2.26 \mathrm{MeV}$, Phys. Rev. $C 84(2011) 022801(\mathrm{R})$.

[6] H. Costantini, R. J. deBoer, R. E. Azuma, M. Couder, J. Görres, J. W. Hammer, P. J. LeBlanc, H. Y. Lee, S. O’Brien, A. Palumbo, E. C. Simpson, E. Stech, W. Tan, E. Uberseder, and M. Wiescher, ${ }^{16} O(\alpha, \gamma){ }^{20} \mathrm{Ne} S$ factor: Measurements and R-matrix analysis, Phys. Rev. C 82 (2010) 035802. 
[7] K. H. Hahn, K. H. Chang, T. R. Donoghue, and B. W. Filippone, Search for nonresonant capture in the ${ }^{16} O(\alpha, \gamma){ }^{20}$ Ne reaction at low energies, Phys. Rev. C 36 (1987) 892-898.

[8] K. Langanke, The ${ }^{16} O(\alpha, \gamma)^{20}$ Ne direct capture reaction at low energies, Z. Phys. A 317 (1984) 325-328.

[9] U. Hager, L. Buchmann, B. Davids, J. Fallis, U. Greife, D. A. Hutcheon, D. Irvine, D. Ottewell, S. Reeve, A. Rojas, and C. Ruiz, Direct measurement of the ${ }^{16} O(\alpha, \gamma){ }^{20} N e$ reaction at $E_{c . m .}=2.43$ and 1.69 MeV, submitted to Phys. Rev. C.

[10] A. Coc, M. Hernanz, J. José, and J.-P. Thibaud, Influence of new reaction rates on ${ }^{18} \mathrm{~F}$ production in novae, Astron. Astrophys 357 (2000) 561-571.

[11] A. Chafa, V. Tatischeff, P. Aguer, S. Barhoumi, A. Coc, F. Garrido, M. Hernanz, J. José, J. Kiener, A. Lefebvre-Schuhl, S. Ouichaoui, N. de Séréville, and J.-P. Thibaud, Experimental determination of the ${ }^{17} \mathrm{O}(p, \alpha){ }^{14} \mathrm{~N}$ and ${ }^{17} \mathrm{O}(p, \gamma){ }^{18} \mathrm{~F}$ reaction rates, Phys. Rev. $\mathrm{C} 75$ (2007) 035810.

[12] C. Fox, C. Iliadis, A. E. Champagne, R. P. Fitzgerald, R. Longland, J. Newton, J. Pollanen, and $\mathrm{R}$. Runkle, Thermonuclear reaction rate of ${ }^{17} O(p, \gamma)^{18} \mathrm{~F}$, Phys. Rev. $\mathrm{C} 71$ (2005) 055801.

[13] J. José and M. Hernanz, Nucleosynthesis in classical novae: CO versus ONe white dwarfs, Astrophys. J. 494 (1998) 680.

[14] C. Iliadis, Art Champagne, Jordi José, Sumner Starrfield, and Paul Tupper, The Effects of Thermonuclear Reaction-Rate Variations on Nova Nucleosynthesis: A Sensitivity Study, Astrophys. J. Suppl. 142 (2002) 105-137.

[15] K. Jayamanna, A multicharge ion source (supernanogan) for the OLIS facility at ISAC/TRIUMF., Rev. Sci. Instrum. 81 (2010) 02A331.

[16] U. Hager, L. Buchmann, B. Davids, J. Fallis, B. R. Fulton, N. Galinski, U. Greife, D. A. Hutcheon, D. Ottewell, A. Rojas, C. Ruiz, and K. Setoodehnia, Measurement of the ${ }^{17} O(p, \gamma){ }^{18} \mathrm{~F}$ reaction rate at astrophysically relevant energies, Phys. Rev. C 85 (2012) 035803.

[17] C. Rolfs, Spectroscopic factors from radiative capture reactions, Nucl. Phys. A 217 (1973) 29-70.

[18] J. R. Newton, C. Iliadis, A. E. Champagne, J. M. Cesaratto, S. Daigle, and R. Longland, Measurement of ${ }^{17} O(p, \gamma){ }^{18} F$ between the narrow resonances at $E_{r}^{l a b}=193$ and $519 \mathrm{keV}$, Phys. Rev. C 81 (2010) 045801 .

[19] G. Lotay, P. J. Woods, D. Seweryniak, M. P. Carpenter, R. V. F. Janssens, and S. Zhu, $\gamma$-ray spectroscopy study of states in ${ }^{27}$ Si relevant for the ${ }^{26} A l^{m}(p, \gamma)^{27}$ Si reaction in novae and supernovae, Phys. Rev. C 80 (2009) 055802.

[20] C. M. Deibel, J. A. Clark, R. Lewis, A. Parikh, P. D. Parker, and C. Wrede, Toward an experimentally determined ${ }^{26} \mathrm{Al}^{m}(p, \gamma)^{27} \mathrm{Si}$ reaction rate in one novae, Phys. Rev. C 80 (2009) 035806. 